Archives de sciences sociales des religions

152 | octobre-décembre 2010

Bulletin Bibliographique

\title{
Pascal Michon, Fragments d'inconnu. Pour une histoire du sujet
}

Paris, Éditions du Cerf, 2010, 251 p.

Daniel Vidal

\section{CpenEdition}

\section{Journals}

Édition électronique

URL : http://journals.openedition.org/assr/22073

DOI : $10.4000 /$ assr.22073

ISSN : $1777-5825$

Éditeur

Éditions de l'EHESS

Édition imprimée

Date de publication : 31 décembre 2010

Pagination : 9-242

ISBN : 9782713223013

ISSN : 0335-5985

\section{Référence électronique}

Daniel Vidal, «Pascal Michon, Fragments d'inconnu. Pour une histoire du sujet », Archives de sciences sociales des religions [En ligne], 152 | octobre-décembre 2010, document 152-88, mis en ligne le 06 mai 2011, consulté le 21 septembre 2020. URL : http://journals.openedition.org/assr/22073 ; DOI : https:// doi.org/10.4000/assr.22073

Ce document a été généré automatiquement le 21 septembre 2020

(C) Archives de sciences sociales des religions 


\section{Pascal Michon, Fragments d'inconnu. Pour une histoire du}

\section{sujet}

Paris, Éditions du Cerf, 2010, 251 p.

\section{Daniel Vidal}

\section{RÉFÉRENCE}

Pascal Michon, Fragments d'inconnu. Pour une histoire du sujet, Paris, Éditions du Cerf, 2010, $251 \mathrm{p}$.

1 Le sujet, cet inconnu. Ou, plus exactement, cela qui a été maintenu hors champ par les sciences sociales et les philosophies contemporaines. Non que les unes et les autres l'aient ignoré, mais il fut sollicité de façon telle qu'il a toujours été soumis, selon P. Michon, à des systèmes référentiels qui en gommaient l'intelligibilité singulière. Sujet à proprement parler "assujetti», quand il convient aujourd'hui, pour l'auteur, philosophe et historien, d'en proclamer la souveraineté critique et la capacité interprétante, ainsi qu'il en allait déjà en ses Éléments d'une histoire du sujet (1999) et sa Poétique d'une anti-anthropologie. L'herméneutique de Gadamer (2000). Et l'analyse démontrera que le sujet, toujours discontinu et en partie aléatoire, «fragmenté », est site de «l'aventure » du vivre, son "advenue ». Site d'une existence singulière, dont Kierkegaard, que curieusement P. Michon n'évoque pourtant jamais, peut être considéré comme l'inventeur, au seuil de nos modernités. La critique adressée ici aux sciences sociales quant à leur prise en charge de la question du sujet est solidement étayée par le constat du primat accordé à l' «absolutisation du social», ultime référentiel invoqué dans le traitement de la subjectivation, ou de la conception de la langue (et non du langage, ce qui change toute la donne de la dispute théorique) comme « fondement ontologique ou noyau de communication intersubjective ». Dans le premier cas, les sciences humaines auraient d'emblée identifié en l'œuvre de Durkheim, 
l'argument qui devait les autoriser à subordonner le sujet à l'inscription en lui des impératifs sociétaux qui l'obligent. On n'en vient au sujet qu'au travers de la pleine intelligence du social. Il en va de même pour la sociologie moderne du langage, qui, exception faite de l'ethnométhodologie - encore que l'auteur demande qu'on l'examine de plus près - considère le langage comme sous-système social, à l'égal d'autres instances ou agences d'historicité. Weber pourrait sans doute être ici convoqué comme au principe de cette prise en compte du langage comme signe ne tenant valeur que de sa combinaison avec tout autre principe d'égale intensité dans la constitution des types idéaux.

2 Du social à la langue, il convient donc, selon P. Michon, de «briser les jeux de miroirs ", afin que de ces éclats puisse se penser véritablement le sujet «radicalement historique ». La philosophie, et le « tournant linguistique » qu'elle accomplit depuis une génération, ne permet pas non plus de poser le sujet en cette radicalité. Sans doute, Heidegger pense-t-il le langage - et non plus la langue - comme site du sujet, lorsque porté à son plus haut niveau d'accomplissement poétique. Mais cela s'entend comme "discours a-logique capable de faire l'expérience de l'Être » - et là encore le poétique même se trouve habilité par une référence en absolu, qui en légitime, en quelque sorte, la possibilité. Mais le langage, et la subjectivité qu'il dénote, ne peuvent s'entendre comme rapportés à des référentiels de surplomb - Être ou société. Ils ne peuvent signifier le sujet que comme «interprétant du social, sans lequel, souligne l'auteur, celui-ci ne saurait fonctionner ni exister ». Il n'est pas d'en-deçà du langage, non plus qu'un au-delà. La «langue» ni la "Tradition» ne fondent l'invention du sujet. Analysant la lecture par Gadamer des poèmes de Paul Celan, P. Michon identifie le point précis où l'interprétation lui semble prendre le pas sur le dit poétique en tant que signifiance en absolu. On pourrait cependant objecter à l'auteur que, loin de proposer une lecture «clinique » de Celan, qui se pencherait au sens propre sur l'œuvre à partir d'une décision en amont, Gadamer tente de ne faire dire au poème rien qui ne soit précisément le « dit » de ce poème. Et seulement ce qu'il dit en vérité. Mais $P$. Michon souligne par là le risque propre à l'herméneutique, de substituer au texte, au langage, au poème, ces analogons que sont le discours commun, la "langue en action", l'écriture saisie en son fil. Et, par là, de rater le sujet, ou la subjectivité, qui fait effraction radicale comme historicité à chaque fois réinventée.

Quant au sujet, précise l'auteur, les sciences sociales se fourvoient en assimilant subjectivation et individuation, ou/et "approfondissement " intérieur. Le reproche paraît avéré si l'on s'en tient aux théories sociologiques fondées sur le paradigme d'un sujet rationnel ajustant son action à un calcul stratégique où doivent s'équilibrer contribution et rétribution. Sujet entièrement «contractuel », en effet, et dont toute subjectivité disparaît comme capacité à surprendre, et suspendre, le monde. Mais les sciences sociales se sont éloignées depuis quelques longues décennies, de cette conception, pour autant qu'elles en aient été en leur principe porteuses. La sociologie de Touraine, par exemple, «pense le sujet » comme acteur ultime de l'historicité, en la radicalité même que sollicite la méditation de P. Michon. Si l'on en vient à des champs sociologiques plus précis, concernant par exemple la «science» des religions, il est clair que la mystique, en son dire et son écriture, et tout réquisit sociologique observé, ne peut s'entendre que comme site du sujet en sa singularité et son rythme propre. Que l'on puisse rabattre cette subjectivité sur des enchaînements de causalités qui en diraient la raison, bien des analystes l'ont tenté. Mais d'autres, auxquels il conviendrait 
d'accorder plein crédit - M. de Certeau par excellence, ou J. LeBrun-, pensent véritablement le «sujet » mystique comme impératif primant sur la raison sociale ou historienne, rejoignant ainsi P. Michon au centre vif de sa quête. Car le dit mystique est bien cette «hyper-expérience du fonctionnement du langage » qui est, pour l'auteur, l'autre nom du sujet. Par lui, comme en poétique, et au rebours de la saisie positiviste de l'action humaine, et du dualisme qui confronte et relie individu et société, le langage n'est plus (seulement) ce qui nous "émancipe des déterminismes ", autorisant ainsi, selon P. Ricœur, qu'une identité se construise. Pas plus qu'un jeu de signes engrenés les uns aux autres "sans jamais permettre à une signification de se fixer ", définissant alors ce qui relève, pour Derrida, spécifiquement de la langue. À l' « interprétance » du social ou de la langue, P. Michon substitue l' «interprétance» du langage. C'est le langage qui dit le sujet, et qui dit la raison, ou la déraison, de la société et de l'histoire. Mais il fait bien plus que la dire : il l'institue. Le sujet est dès lors l'instituant de l'existence, singulière aussi bien que collective. Bref, le sujet est l'absolu du regard et de la voix.

"Sujet et langage sont des inconnus que nous ne pouvons saisir que par fragments", écrit P. Michon. Il lui faut alors examiner toutes les ressources philosophiques, de Hegel et Kant à Nietzsche et Foucault, pour en venir à cette mise en question et en cause des significations de notre héritage des Lumières et de notre modernité. Que la modernité hégélienne s'entende comme liberté de la subjectivité en même temps qu'éclatement de la raison; que le jeu circulaire de l'herméneutique gadamérienne suppose le primat de la langue et de la Tradition; que la communication intersubjective, chez Rawls ou Arendt, requière l'analyse des transcendantaux; que tout discours, chez Nietzsche, Foucault ou Deleuze, soit lieu de « diffèrement transversal » fondant « l'agonistique de la communication »- l'ensemble de ces œuvres, qui ne se libèrent que partiellement de la conception d'un "sujet substantiel traditionnel ", ne paraissent plus à même de penser le sujet en sa radicalité historique. On a vu qu'il fallait pour cela fonder le langage comme seule modalité du sujet, et le langage porté à son excès et sa plus haute vertu, et vérité : le poème. P. Michon revisite alors Kant et le " jugement esthétique »; Gadamer et, nous l'avons vu, son questionnement sur l'art, qui ouvre Vérité et Méthode, etc. Ce furent des annonciateurs qui cependant, selon l'auteur, ne rompirent pas catégoriquement avec les cadres a priori du sujet et du langage.

5 Avec Simmel et, plus directement, avec Groethuysen, l'homme «se problématise continûment» à partir des "pratiques sémantiques» (et non sémiotiques, qui renverraient à la langue, et non au langage) qui « soutiennent cette problématisation ». L'ouvrage traitera alors de ce langage/sujet dans les termes de l'art. Il faut, écrit P. Michon, "se retourner vers une véritable poétique de l'art "s'inscrivant «en faux contre l'idée d'un éclatement irrémédiable des sphères de valeurs (...) Une telle expérience (esthétique) est toujours à la fois cognitive, éthique et politique, car elle est toujours expérience de subjectivation ». Art, langage, sujet, topiques équivalentes. Disant l'un, nous énonçons l'autre, en une passion unifiante qui fait de l'ouvrage de P. Michon un brûlot contre le dualisme et sa tradition socio-philosophique. Penser le sujet est ainsi penser ce qui excède la langue et l'histoire, la modernité et la postmodernité. Car c'est leur substituer «le thème pluraliste mais non relativiste d'un infini de modernités apparaissant continuellement au cours de l'histoire des hommes ». Le langage est ainsi cette "activité » irradiante et interprétante qui est le seul lieu du sujet. Humboldt le plaçait «au fondement de l'historicité humaine ", non pas comme principe de potentialité, mais, selon la terminologie de P. Michon, d' «instanciation ». 
Non pas puissance de, mais accomplissement en soi. Et donc, instance d'emblée d'historicité, en son « instant » même. Commentant Humboldt, E. Cassirer notait avec perspicacité qu'il cherchait « à parvenir au point où l'opposition de la subjectivité et de l'objectivité, de l'individualité et de l'universalité se résout pour devenir pure indifférenciation ». Est-on si éloigné du « sentiment océanique » freudien, ou du pari en absolu du surréalisme? Loin, en tout cas, de ne se penser que sous condition de référence à l'Être, comme il en va chez Heidegger, le langage, selon la belle formule de l'auteur, est la «maison de l'être » - et sa raison. L'arbitraire du signe, chez Saussure, concerne langue plus que langage. C'est de cet «arbitraire » que la langue tient son statut de contrat inter-personnel. Mais cette convention, "aléatoire et immuable", régit des types d'historicités ( «action du temps [combinée] avec celle des forces sociales", écrit le linguiste). Ainsi se définit une tension entre "l'activité suiréférentielle du langage et son rapport au monde », rapport qu'assume, proprement dit, la langue. Aussi bien l'auteur peut-il conclure avec Saussure, en la «radicale historicité des systèmes linguistiques ».

6 Mais, dans le mesure où «l'activité du langage» prime absolument sur les modalisations singulières de la langue, P. Michon s'écarte de l'argumentation du linguiste pour "penser l'historicité de l'action [du langage] sans avoir recours à son identification à une action de la collectivité » - ou individuelle. Le langage/sujet prime exactement sur les conditions de l'action en situation et le fil du discours qui s'y tient. De là, sans doute, les réserves que P. Michon exprime à l'égard de l'ethnométhodologie. Benveniste, enfin, pour clore ce passage en revue du statut du langage. Celui-ci est bien le "lieu effectif de signifiance, sans transcendance ", où subjectivation et "sociation " ne se distinguent et moins encore ne s'opposent; où le sujet, le langage et le rapport au monde qu'il(s) inaugure(nt), constituent une seule et même autoréférence. Autrement dit, écrit P. Michon, "chaque acte de langage est une action sociale par laquelle se réinventent sans cesse les relations entre les hommes, et donc le sujet ». Pas plus qu'il n'est soumis au primat du social, le langage n'est assujetti à l'histoire : «c'est le langage qui fonde notre historicité ». Qu'est-ce alors un sujet? "L'émergence dans l'être, commente P. Michon, d'une propriété fondamentale du langage»: le «je», «l'extraordinaire à l'état pur, naissant sans cesse au sein du plus banal ». Le «banal» comme site de transcendance? Un «je» qui «ne reçoit sa réalité que de son énonciation ». Très proches ici sommes-nous sans doute de l'analyse par M. de Certeau du «volo» triomphant de la mystique, où le «je» s'indique comme pure volonté. Benveniste: "Tout homme invente sa langue et l'invente toute sa vie. Et tous les hommes inventent leur propre langue sur l'instant et chacun d'une façon distinctive, et chaque fois d'une façon nouvelle ».

7 Le sujet est ainsi cette invention proprement inouie qui vient avec le langage, ainsi que chez Rimbaud la mer allée avec le soleil. Penser langage est penser sujet. P. Michon : «Le langage n'est ni principe de liberté, ni condition de possibilité, ni nécessité, ni horizon, ni chaos. Il est de l'ordre d'une forme de ce qui est mouvant, d'un rythme et d'une manière de fluer». Ainsi en va-t-il du sujet, que l'auteur peut alors mettre «à l'épreuve de la littérature ». L'auteur reproche à la critique herméneutique du sujet de dissocier le sens de la forme. Ainsi s'oppose-t-il à la lecture par Gadamer de la poétique de Celan, qui privilégierait le sens au détriment du dit poétique en sa forme pure. Le lecteur peut avoir quelque réticence à admettre le bien-fondé de cette critique: Gadamer pose en effet l'acte de lecture comme inscrit au principe de l'œuvre, comme participant de son efficacité symbolique et éthique, et de son historicité. Bref, de la 
subjectivité qui s'y joue. Sans préjuger, au contraire de ce qu'écrit P. Michon, de référence à quelque "sens caché ou perdu». Au demeurant, l'auteur en pourrait convenir, qui définit le langage comme "activité productrice de signifiance et de transsubjectivité, et condition de possibilité d'une production infinie de formes de vie nouvelles - une infinité de formes de subjectivation - une infinité de modernités». Ainsi en va-t-il du sujet, cette "émergence liée au langage comme activité » - sujet aussitôt pluraliste, historié, abolis tous les garants méta-sociaux de l'ordre social. En ce point précis, l'œuvre de Meschonnic est à juste titre sollicitée. Car pour l'auteur de la Critique du rythme. Anthropologie historique du langage (1982) et de Politique du rythme, politique du sujet (1995), le sujet n'est « ni substance, ni forme, ni nœud de forces, mais instanciation du langage ». Non qu'il soit immanent à celui-ci, mais il en est, si l'on peut dire, le for intérieur - cette intimité habitée jusqu'à faire présence pure. L'art, ce langage porté à sa plus haute tension, et énergie, et le sujet qui s'y livre selon son rythme propre, est ainsi à la fois «individu», absolument singulier, et "unité ouverte", "à l'origine d'une chaîne infinie de ré-énonciations». Telle est bien l'épreuve de la littérature, à laquelle P. Michon soumet son théorème du sujet. Le " je " n'est jamais que cet autre dont Rimbaud a proclamé l'éclat premier, dans le sillage de Hugo (" Ô insensé qui crois que je ne suis pas toi), et en invite à Aragon («"je" exprime tout le concret de l'homme »). Et le sujet, par cette concrétude même, par cette altérité instituante, par cette vocation à universalité, assume ainsi un double défi : n'être qu'à lui-même sa propre référence, et, selon son rythme et sa passion du temps, prendre l'histoire à pleine voix, et, prophétique et utopique, disant l'aventure du monde et «maximisant la puissance du vivre». Ainsi est-il en son langage l'interprétant "poétique » du social en la pluralité de ses significations, la discontinuité de son histoire, et la mobilité de ses accomplissements. Le sujet, ou le signifiant donnant « chair sémantique historique » au social. Mais sujet aussi bien, l'œil fixant Caïn en sa tombe. 